\title{
Ternary and Higher Order Aluminum Phase Diagram Updates
}

Updates on ternary and higher order aluminum phase diagrams are published regularly in this journal. These brief reviews include new phase diagrams of systems that are investigated for the first time, as well as previously-investigated systems on which additional information or substantial revision of existing data has become available.

This issue carries updates on eight ternary systems: Al-Co-Cu, Al-Cr-Ti, Al-Mn-Si, Al-Nb-Ti, Al-Pd-Ti, Al-Pt-Ti, Al-Sb-Zn and Al-Ti-V and the quaternary system: Al-Cr-Ti-V.

This work was supported by the Indian Institute of Metals, Calcutta.

V. Raghavan

Editor,

Phase Diagrams of Ternary Iron Alloys

Parts 1, 2, 3, 5, and 6 